\title{
Berufsorientierung in Moscheen
}

\section{Erfahrungen aus einem Projekt der Jugendsozialarbeit}

Alois Becker

Dr. Alois Becker ist Direktor der

Akademie Klausenhof am Niederrhein.

Die Weiterbildungseinrichtung

(Heimvolkshochschule) begann ihre

Arbeit 1959 als zentrale Bildungsstätte

der Katholischen Landjugendbewegung

(KLJB). Heute umfasst ihr Angebot alle

Bereiche der Weiterbildung mit den

Schwerpunkten Migrantenarbeit sowie

Arbeit und Beruf.

Internet:

http://www.akademie-klausenhof.de
Die Ausbildungsquote von Jugendlichen mit Migrationshintergrund ist niedrig und sinkt in den letzten Jahren sogar noch weiter. In Nordrhein-Westfalen wurde deshalb der Versuch unternommen, mit Informationsveranstaltungen in Moscheen junge Menschen und ibre Angehörigen auf die Bedeutung und die Möglichkeiten beruflicher Qualifizierung hinzuweisen.

Im Sommer 2007 machte der Bundestagsabgeordnete Uwe Schummer (CDU) in einer Pressemitteilung zur Ausbildungssituation den auf den ersten Blick ungewöhnlichen Vorschlag, doch in den Moscheen für bessere Bildung und mehr Ausbildungsbereitschaft zu werben. Bei genauerem Nachdenken besticht diese Idee aber durchaus. Zwar können dort wahrscheinlich weniger die Jugendlichen angesprochen werden, aber sicher die für den Lebensweg entscheidenden Familienangehörigen: Väter, Onkel, leider kaum die Mütter. Aber wie kommt man in die Moscheen hinein? Und woher sollen wir erfahren, wo sich welche Moschee von welchem Verein befindet? Und wie knüpft man Kontakte mit dem jeweiligen Imam und wie steht der überhaupt zu einer solchen Initiative?

Nicht erst seit PISA wissen wir von der schwierigen Situation der Jugendlichen mit Migrationshintergrund beim Übergang von Schule in die Arbeitswelt. Manchmal ist man beim Lesen älterer Aufsätze und Papiere schon erstaunt, wie aktuell sie sind, lässt man Spracheigenheiten und Jahreszahlen weg. Aktuell geht man von rund zwanzig Prozent jugendlichen Migranten und Migrantinnen aus, die die Schule ohne Abschluss verlassen, hinzukommen noch diejenigen mit schwachen Ergebnissen vor allem in Deutsch und Mathematik. So kann es nicht verwundern, wenn die Ausbildungsquote bei dieser Personengruppe nur zwischen 25 und 30 Prozent liegt. Diese Quote geht sogar seit einigen Jahren zurück. Zum Vergleich: Bei deutschen
Jugendlichen liegt die Quote bei 60 bis 65 Prozent. Zur schlechten schulischen Ausgangsituation kommen noch weitere Faktoren hinzu wie falsche Vorstellungen über die duale Berufsausbildung, die sich in mangelnder Motivation oder eingeschränkter Berufswahl niederschlagen.

Im Projekt »Lernender Niederrhein Übergänge und Brücken « (Programm des Bundesministeriums für Bildung und Forschung: "Lernende Regionen - Vertiefungsphase I2) sollte diese Gruppe daher besonders berücksichtigt werden. Um in die Thematik einzuführen, wurden zu den beiden zentralen Auftaktveranstaltungen der Integrationsbeauftragte der Landesregierung Nordrhein-Westfalen, Thomas Kufen und Bülent Arslan vom »Institut für interkulturelle Managementund Politikberatung - IMAP « eingeladen. Die inhaltliche Projektarbeit legte den Fokus zunächst auf die Sammlung aller Aktivitäten im Bereich des Übergangs von Schule zu Beruf, um den beteiligten Akteuren einen Überblick über bereits existierende Möglichkeiten und Maßnahmen zu verschaffen. Beteiligte sind in diesem Feld vor allem die Beratungslehrkräfte der Hauptschulen und Berufskollegs (Berufsschulen), Beratungsstellen, Maßnahmeträger, Kommunen und die Organisationen der Wirtschaft. Die Vielfalt der zutage geförderten Beispiele ist erstaunlich und überrascht sogar die Fachleute. Als Ergebnis entstand ein Wegweiser für die Kreise Kleve und Wesel sowie ein Sprachkurskompass.

In Kooperation mit der Kreishandwerkerschaft, der Niederrheinischen Industrie- und Handelskammer und dem lokalen Rundfunksender »Radio K.W.« entwickelten wir eine Kampagne mit Werbespots, informativen redaktionellen Kurzbeiträgen sowie einem Tag der Ausbildung mit Experten im Studio. Diese Aktivität fand kreisweit eine große Resonanz. Diese Informationen sind für alle Jugendlichen bedeutsam, unabhängig von ihrer Nationalität oder Herkunft. 


\section{Vorbereitung des Tages der Ausbildung in den Moscheen}

Bedingt durch die Standorte DinslakenLohberg und Kamp-Lintfort der Ruhrkohle AG und von Großbetrieben der Metallverarbeitung (Babcock) sind die Türken die größte Gruppe von Migranten. Deshalb haben wir uns aufgrund der kurzen Laufzeit des Projekts (18 Monate) und der beschränkten Mittel auf diese Gruppe konzentriert, obwohl im Raum Kleve die aus dem Gebiet der GUS-Staaten Zugezogenen eine stärkere Population darstellen und sich der Übergang der Jugendlichen in die Arbeitswelt ebenfalls nicht einfach gestaltet.

Benötigt wird ein »Türöffner « oder Scout, der durch diese fremde und unbekannte Welt führt. Wir fanden ihn mit dem erwähnten IMAP-Institut, das auch die bekannte Kopftuch-Studie für die Konrad-Adenauer-Stiftung verfasst hat. schneller, nach der Veranstaltung in Friedrichsfeld sprach uns der Vorsitzende einer zweiten Moschee in Voerde an, weil er auch gerne eine solches Angebot machen wollte.

Nachdem die Veranstaltungen in den Moscheen beendet waren, meldete sich ein örtlicher Integrationsrat, mit dem Wunsch eine solche Veranstaltung auch in Kooperation mit ihm durchzuführen, da wir dann einen weiteren Personenkreis ansprechen könnten.

\section{Der Ablauf der Veranstaltungen}

Die Veranstaltung wurde einige Wochen vor dem Termin am Ende der Freitagsgebete angekündigt, unterstützt durch türkischsprachige Flyer und einem Plakat. Die Netzwerkpartner wurden frühzeitig über das Vorhaben informiert und um Unterstützung gebeten. Bei den

\section{»Obne einen >Türöffner läuft in Moscheegemeinden wenig "}

Die vorgeschlagene Verfahrensweise: Am besten eignet sich ein Termin im Anschluss an das Freitagsgebet, da dies erfahrungsgemäß die meist besuchte Veranstaltung ist.

Nach der Identifizierung von gesprächsbereiten Moscheen sollten Vorbereitungstreffen stattfinden, auf denen der weitere Ablauf abgesprochen werden sollte. Das erste konkrete Gespräch führten wir nach einem Freitagsgebet in der Moschee in Dinslaken-Lohberg, und zwar mit dem ehrenamtlichen Vorstand des Moscheevereins. Die Aufnahme war sehr freundlich, aber zuerst noch zurückhaltend. Aber nach Darstellung unseres Vorhabens schwand die anfängliche Skepsis zunehmend und wir wurden uns über das weitere Vorgehen einig. Freilich gab es zeitliche Hindernisse: erst müsse das Einverständnis des Imam eingeholt werden, die Sommerferien und dann die Fertigstellung des Umbaus abgewartet werden, während des Ramadan geht es nicht, also ein Termin im Oktober! In der zweiten Moschee (Voerde-Friedrichsfeld) ein ähnlicher Ablauf, allerdings ohne Umbauprobleme. Auch hier ein Termin erst im November. Bei der dritten ging es
Veranstaltungen selbst wirkten die Agentur für Arbeit Wesel (Berufsberater für Migranten), die Handwerkskammer Düsseldorf, ein Vertreter der Akademie Klausenhof/Lernende Region, das IMAP-Institut in vorsorglich bereitgestellter Dolmetscherfunktion und ein Vertreter der Polizei Kreis Wesel mit. Dort hatte der sogenannte Islambeauftragte von unseren Veranstaltungen durch die Plakate erfahren und bat um Mitwirkungsmöglichkeit, da die Polizei derzeit verstärkt Bewerberinnen und Bewerber mit Migrationshintergrund suche.

In Eingangsstatements wurde auf die Bedeutung der beruflichen (dualen) Ausbildung auch für Deutsche hingewiesen, Wege aufgezeigt, wie an Ausbildungsplätze zu kommen ist; Möglichkeiten dargestellt, was getan werden kann, wenn die Schule ohne oder mit schlechtem Abschluss beendet wird. Im Anschluss kamen die Teilnehmenden zu Wort. Die Veranstaltungen dauerten etwa anderthalb bis zwei Stunden und endeten mit der Bitte um weitere Kontakte.

Teilnehmende waren ungefähr je zur Hälfte ältere männliche Erwachsene (Vereinsvorstände, Eltern, Verwandte) und betroffene männliche Jugendliche; in Dinslaken-Lohberg zusammen rund 30, in Voerde 20 und 15 Teilnehmende. Eine Übersetzung wurde kaum benötigt, die Erwachsenen verstanden, was gemeint war, wobei sich nur die zu Wort meldeten, die sich in Deutsch gut ausdrücken konnten. Die Jugendlichen hatten keinerlei Verständigungsprobleme, auch wenn ihr Deutsch manchmal das gern karikierte Türkisch-Deutsch war. Anderseits verstärkten die Einlassungen des IMAP-Vertreters die Aussagen der deutschen Experten.

\section{Ergebnisse und Erkenntnisse}

Die Vorstände der Moscheevereine zeigten sich nach anfänglicher Zurückhaltung sehr interessiert und betonten, wie wichtig sie eine berufliche Integration der Jugendlichen halten. Ob diese positive Haltung auf eine vorige »Bearbeitung « durch das IMAP-Institut zurückzuführen ist, lassen wir dahingestellt. Auf jeden Fall sind diese Vereine wichtige Ansprechpartner, denn in den größeren Vereinen mit entsprechenden Räumlichkeiten organisieren sie auch Bildungsveranstaltungen und bieten Freizeitgestaltung an, gerade auch für Jugendliche. Man könnte sie also, auch wenn der Vergleich hinken mag, mit den Pfarr- oder Kirchengemeinden in den christlichen Kirchen vergleichen.

Die Väter machten einen sehr nachdenklichen, manchmal bedrückten Eindruck, wenn sie über das Verhalten und die Zukunftsaussichten ihrer Kinder, insbesondere der Söhne sprachen. Eine gewisse Hilflosigkeit war nicht zu verkennen. Vor der Schließung der Zeche oder stahlverarbeitender Betriebe besorgten die Väter den Söhnen (wie auch bei deutschen Arbeitern üblich) eine Stelle im eigenen Betrieb, was nun nicht mehr möglich ist. Welch seltsame Blüten diese Haltung treibt, drückte ein jüngeres Vorstandsmitglied (außerdem Betriebsratsmitglied) aus so aus: »Vermutlich aus einer Mischung aus Unterstützungswille und Hilflosigkeit gäben die Väter ihren Jugendlichen ohne Arbeit pro Tag zehn Euro Taschengeld. Mit etwas Jobben hie und da kämen die jungen Kerle sehr gut über die Runden. « Kein Wunder, wenn sie dann dauernd in Cafés säßen oder mit aufgemotzten Autos herumführen. Jeden- 
falls sei der Anreiz, eine Berufsausbildung zu beginnen, sehr gering.

Bekannt ist die Unkenntnis der Väter und der Jugendlichen über das deutsche System der dualen Ausbildung. Wenn eine Ausbildung in Frage kommt, dann eine schulische, aber ohne nun recht $\mathrm{zu}$ wissen, was welche Schulform wirklich bringt. Da auch die Beratungslehrer hier nicht immer über genügend Wissen verfügen, landen die Jugendlichen nach Ende der allgemeinen Schulpflicht in irgendeiner Schulform der Berufskollegs (Berufsschulen); oft dort, wo gerade ein Platz frei ist. Verbunden damit ist die trügerische Hoffnung, in diesen Schulformen den bisher nicht erreichten Hauptschul- oder mittleren Bildungsabschluss so im Vorübergehen zu bekommen. Angestrebt wird aber eigentlich eine ordentlich bezahlte (Hilfs-) Arbeit. Berufsausbildung ist in diesem Sinn eine schlecht bezahlte Tätigkeit, keine Investition in die $\mathrm{Zu}$ kunft. Nicht selten werden trotzdem gewünschte Tätigkeiten genannt, für die ein Studium Voraussetzung ist.

Erschreckend war die Tatsche, dass nur ganz wenige der anwesenden Jugendlichen sich bei der Agentur für Arbeit als Ausbildungsplatz suchend gemeldet hatten, obwohl sie mit ihren Schulklassen das Berufsinformationszentrum besuchten und auch Kontakte zur Berufsberatung vorhanden waren.

Erfreulich war die Resonanz auf die Anwesenheit der Polizisten (wir hatten zunächst große Zurückhaltung befürchtet) und das Interesse an der Möglichkeit auch ohne deutsche Staatsangehörigkeit dort eine Ausbildung machen zu können. Allerdings liegt die Messlatte in Nordrhein-Westfalen mit der (Fach-) Hochschulreife sehr hoch. Die Botschaft kam jedoch an.

Sehr positiv aufgenommen wurde die Tatsache, dass sich (deutsche) Experten erstmalig auf den Weg machten und die anzusprechenden Menschen (»Problemgruppe des Arbeitsmarktes «) in der Moschee aufsuchten.

\section{Schlussfolgerung}

Auch wenn unsere Aktion nur ein Versuch war, ob eine solche Strategie weiterführen könnte, waren wir doch angenehm über das Interesse überrascht. Leider war es wegen der begrenzten Laufzeit des Projektes (Ende 31.12.2007) und der benötigten Vorlaufzeit nicht mehr möglich, weitere Moscheen zum Beispiel im linksrheinischen Teil des Niederrheins etwa in Moers oder Kamp-Lintfort mit hohem Anteil türkischstämmiger Bevölkerung aufzusuchen. Ebenso konnten wir keine Erfahrung mit Moscheen oder Moscheevereinen sammeln, die anderen Dachverbänden als der Türkisch-Islamischen Union der Anstalt für Religion e. V. (DITIB) angehören wie etwa dem Verein islamischer Kulturzentren (VIKZ). Dennoch sind wir überzeugt, dass dieser Weg Erfolg versprechend ist. Die Anfragen aus bislang nicht angesprochenen Moscheen oder dem zitierten Integrationsrat zeigen dies.

Auch die bei unseren (deutschen) Partnern anfänglich vorhandene Skepsis hat sich gelegt. Moscheen erscheinen ja wie eine ferne, sehr fremde Welt, die zudem in der Öffentlichkeit in Fundamentalismusverdacht stehen. Ein schneller Erfolg wird freilich auch bei vielen Veranstaltungen in Moscheen nicht zu erzielen sein. Aber wie bei der legendären Aktion Freiburger Studenten "Student aufs Land " in den1960er Jahren könnte die Langzeitwirkung erstaunlich sein.

\section{"Einer der besten seiner Zunft.»}

Prof. Dr. H.-D. Gottlieb, in: Sozialrecht aktuell 1/99

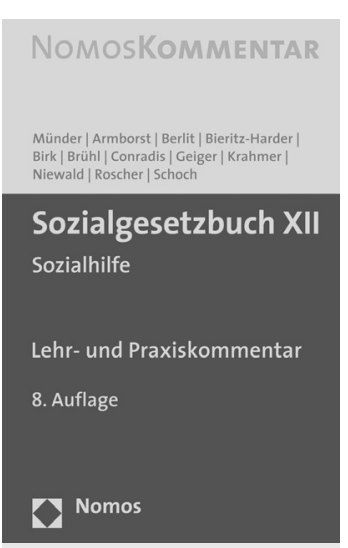

Sozialgesetzbuch XII

Sozialhilfe

Lehr- und Praxiskommentar

Von Prof. Dr. Johannes Münder, MinR Christian Armborst, RiBVerwG Prof. Dr. Uwe Berlit, Prof. Dr. Renate Bieritz-Harder, Prof. Dr. Ulrich-Arthur Birk, Prof. Dr. Albrecht Brühl, RA Dr. Wolfgang Conradis, FASozR, RiSG Udo Geiger, Prof. Dr. Utz Krahmer, RiLSG Stephan Niewald, Prof. Dr. Falk Roscher und RegDir a.D. Dietrich Schoch

8. Auflage 2008, 1.017 S., geb., 44,- $€$, ISBN 978-3-8329-2930-5

Die Neuauflage des LPK-SGB XII bringt Sie im Sozialhilferecht wieder auf den neuesten Stand.

"Ein hochaktuelles und kompaktes Werk zu einem fairen Preis." Ulrich Harmening, Zeitschrift für das Fürsorgewesen 8/05

"Auf diesen angesichts seines Umfangs und seiner Qualität verhältnismäßig sehr preiswerten Lehr- und Praxiskommentars sollte keine Sozialberaterin und kein Sozialberater verzichten." Recht-Informationsdienst der Zeitschrift Caritas in NRW 2/08

Bitte bestellen Sie bei Ihrer Buchhandlung oder bei Nomos | Telefon 07221/2104-37 | Fax -43 | www.nomos.de | sabine.horn@nomos.de 
„Die meisten Opfer islamistischen Terrors sind Moslems." Jürgen Becker, deutscher Fernsehmoderator (geb. 1959)

"Toleranz sollte eigentlich nur eine vorübergehende Gesinnung sein; sie muss zur Anerkennung führen. Dulden heißt beleidigen." Johann Wolfgang von Goethe, deutscher Dichter (1749-1832)

"Eine Religion, die den Menschen finster macht, ist falsch." Immanuel Kant, deutscher Philosoph (1724-1804)

„Ist es nicht sonderbar, dass die Menschen so gerne für die Religion fechten, und so ungerne nach ihren Vorschriften leben?"

Georg Christoph Lichtenberg, deutscher Naturwissenschaftler und Schriftsteller (1742-1799)

"Das Beste an der Religion ist, dass sie Ketzer schafft."

Ernst Bloch, deutscher Philosoph (1885-1977)

„Die Zuversicht, mit der ich die Religion eines anderen als Unsinn erkenne, lehrt mich auch meiner eigenen gegenüber skeptisch zu sein." Mark Twain, amerikanischer Schriftsteller (1835-1910)

"Du sollst dich einmischen, wo Toleranz unmoralisch ist."

Ludwig Marcuse, deutscher Schriftsteller (1894-1971) 